\title{
The effectiveness of an online abstract checker application
}

\begin{abstract}
Writing an abstract is difficult for students and they need a tool to check their abstracts against a confirmed framework. The current paper is a report on the usefulness of the online abstract checker application to the users. The application 'abstract checker 1.0' was developed based on Santos' (1996) Framework for writing a successful scientific abstract. The efficacy of the application was verified in a trial session which saw the participation of 32 postgraduate students from different faculties in Universiti Putra Malaysia (UPM). During the session, participants were given instructions on how to use the tool and their feedback on the performance of the application was collected through a questionnaire and notes from mutual talks with the researchers. The special features of the application are the highlighting of the sentences according to the moves and the Abstract Quality Index (AQI) as an indicator of the level of quality of the submitted abstract. The usefulness of the software was analyzed based on a five point Likert scale and the obtained score showed that generally students found the application very useful. However, more importantly, the feedback received from the participants were invaluable information for the researchers to further fine tune the software for a more efficient and trustworthy application.
\end{abstract}

Keyword: Abstract checker; Abstract Quality Index; Applied linguistics; Computer science 